\title{
A Molecular Ruler for Chain Elongation Catalyzed by Octaprenyl Pyrophosphate Synthase and Its Structure-Based Engineering To Produce Unprecedented Long Chain trans-Prenyl Products
}

\author{
Rey-Ting Guo, ${ }^{\S, l, \perp}$ Chih-Jung Kuo, ${ }^{\perp, @ ~ T z u-P i n g ~ K o, ~}{ }^{@}$ Chia-Cheng Chou, ${ }^{@, \# ~ P o-H u a n g ~ L i a n g, ~},,,, \|, @$ and \\ Andrew H.-J. Wang*,§,I,@,\# \\ Taiwan International Graduate Program, Academia Sinica, Taipei 115, Taiwan, Institute of Biochemical Sciences, \\ National Taiwan University, Taipei 106, Taiwan, and Institute of Biological Chemistry and Core Facility for \\ Protein X-ray Crystallography, Academia Sinica, Taipei 115, Taiwan
}

Received December 31, 2003; Revised Manuscript Received March 26, 2004

\begin{abstract}
Octaprenyl pyrophosphate synthase (OPPs) catalyzes consecutive condensation reactions of farnesyl pyrophosphate (FPP) with five molecules of isopentenyl pyrophosphate (IPP) to generate $\mathrm{C}_{40}$ octaprenyl pyrophosphate (OPP) which constitutes the side chain of menaquinone. We have previously reported the X-ray structure of OPPs from Thermotoga maritima, which is composed entirely of $\alpha$-helices joined by connecting loops and is arranged with nine core helices around a large central cavity [Guo, R. T., Kuo, C. J., Ko, T. P., Chou, C. C., Shr, R. L., Liang, P. H., and Wang, A. H.-J. (2004) J. Biol. Chem. 279, 4903-4912]. A76 and S77 are located on top of the active site close to where FPP is bound. A76Y and $\mathrm{A} 76 \mathrm{Y} / \mathrm{S} 77 \mathrm{~F}$ OPPs mutants produce $\mathrm{C}_{20}$, indicating that the substituted larger residues interfere with the substrate chain elongation. Surprisingly, the A76Y/S77F mutant synthesizes a larger amount of $\mathrm{C}_{20}$ than the A76Y mutant. In the crystal structure of the A76Y/S77F mutant, F77 is pushed away by Y76, thereby creating more space between those two large amino acids to accommodate the $\mathrm{C}_{20}$ product. A large F132 residue at the bottom of the tunnel-shaped active site serves as the "floor" and determines the final product chain length. The substitution of F132 with a small Ala, thereby removing the blockade, led to the synthesis of a $\mathrm{C}_{50}$ product larger than that produced by the wild-type enzyme. On the basis of the structure, we have sequentially mutated the large amino acids, including F132, L128, I123, and D62, to Ala underneath the tunnel. The products of the F132A/L128A/I123A/D62A mutant reach $\mathrm{C}_{95}$, beyond the largest chain length generated by all known trans-prenyltransferases. Further modifications of the enzyme reaction conditions, including new IPP derivatives, may allow the preparation of high-molecular weight polyprenyl products resembling the rubber molecule.
\end{abstract}

A class of prenyltransferases synthesizes linear isoprenyl polymers via consecutive condensation reactions of varying numbers of isopentenyl pyrophosphates (IPPs) ${ }^{1}$ with a single farnesyl pyrophosphate (FPP) (1). These prenyltransferases were classified as cis or trans according to the stereochem-

$\dagger$ This work was supported by grants from Academia Sinica and National Science Council (NSC91-3112-P-001-019-Y) to A.H.-J.W. and (NSC92-2113-M-001-026) to P.-H.L.

The atomic coordinates and structure factors (1VG2 for A76Y, 1VG3 for A76Y/S77F, 1VG4 for F132A/L128A, 1VG6 for F132A/ L128A/I123A, and 1VG7 for F132A/L128A/I123A/D62A) have been deposited in the RCSB Protein Data Bank.

* To whom correspondence should be addressed: Institute of Biological Chemistry, Academia Sinica, 128 Academia Road, Taipei 115, Taiwan. A.H.-J.W.: e-mail, ahjwang@gate.sinica.edu.tw; telephone, +886-2-2788-1981; fax, +886-2-2788-2043. P.-H.L.: e-mail, phliang@gate.sinica.edu.tw; telephone, +886-2-2785-5696 ext. 6070; fax, +886-2-2788-9759.

$\S$ Taiwan International Graduate Program, Academia Sinica.

" National Taiwan University.

${ }^{\perp}$ These authors contributed equally to this work.

@ Institute of Biological Chemistry, Academia Sinica.

\# Core Facility for Protein X-ray Crystallography, Academia Sinica. istry of double bonds generated during IPP condensation (2). The cis-prenyltransferases generally make larger products, $\mathrm{C}_{55}-\mathrm{C}_{120}(3-5)$. In an extreme case, natural rubber containing thousands of IPP units is synthesized by cis-type rubber prenyltransferases $(6,7)$. On the other hand, trans-prenyltransferases generate shorter products, including a $\mathrm{C}_{50}$ polymer by decaprenyl pyrophosphate synthase ( 8 ). We had determined the structures of both cis-type undecaprenyl pyrophosphate synthase (UPPs) and trans-type octaprenyl pyrophosphate synthase (OPPs), which provide the mechanisms for determining the $\mathrm{C}_{55}$ and $\mathrm{C}_{40}$ product chain lengths,

\footnotetext{
${ }^{1}$ Abbreviations: IPP, isopentenyl pyrophosphate; FPP, farnesyl pyrophosphate; FPPs, farnesyl pyrophosphate synthase; OPP, octaprenyl pyrophosphate; OPPs, octaprenyl pyrophosphate synthase; SPPs, solanesyl pyrophosphate synthase; DPPs, decaprenyl pyrophosphate synthase; GGPPs, geranylgeranyl pyrophosphate synthase; UPPs, undecaprenyl pyrophosphate synthase; UPP, undecaprenyl pyrophosphate; Tris, tris(hydroxymethyl)aminomethane; Hepes, 4-(2-hydroxyethyl)-1-piperazineethanesulfonic acid; EDTA, ethylenediaminetetraacetic acid; SDS-PAGE, sodium dodecyl sulfate-polyacrylamide gel electrophoresis; PEG, polyethylene glycol; MIR, multiple isomorphous replacement; MR, molecular replacement.
} 


\begin{tabular}{ll}
\hline \multicolumn{1}{c}{ Table 1: Primers Used To Construct OPPs Mutants from Their Preceding Mutants in This Study ${ }^{a}$} \\
\hline mutant & primer sequence \\
\hline F132A/L128A & 5'-GATAGGAAATAACAAAGCGAGAAGAGCTGCTTTGAATGTGATCGGG-3' \\
F132A/L128A/I123A & 5'-GCGTTCCATACGGTTGAAGAGGCAGGAAATAACAAAGCGAGA-3' \\
F132A/L128A/I123A/D62A & 5'-GGAGTAGAGATCGGTGAAGCTGCGATATCTTCTCTTGCAGCT-3' \\
\hline
\end{tabular}

${ }^{a}$ The underlined residues represent the mutagenic sites where the nucleotide sequences were changed.

respectively, by these two enzymes (9-13). Despite the completely different structural folds, both enzymes contain a tunnel-shaped crevice as the active site to accommodate the long chain product. At the bottom of their active site, a large hydrophobic amino acid (L137 for UPPs and F132 for OPPs) serves as the "floor" to block further chain elongation of the correct product. By substitution of L137 in UPPs or F132 in OPPs with small Ala to remove the floor, the chain length is extended to mainly $\mathrm{C}_{75}$ in UPPs and $\mathrm{C}_{50}$ in OPPs $(9,13)$.

Compared to the large polymers, especially natural rubber, made by cis-prenyltransferases, trans-type enzymes generate shorter products (14). In the crystal structure of OPPs, several large amino acids, including Leu128, Ile123, and Asp62, were found underneath F132, which could provide additional blockage at the bottom of the active site. In this study, we have sequentially replaced these amino acids with smaller Ala residues and examined the product chain lengths made by the mutants. We have also determined the threedimensional (3D) crystal structures of these mutants in an effort to provide the structural explanation for the unprecedented product chain length synthesized by the transprenyltransferases.

Wild-type OPPs from Thermotoga maritima catalyzes the chain elongation of FPP to $\mathrm{C}_{40}$ octaprenyl pyropohphate that constitutes the side chain of menaquinone, an essential component involved in the electron transfer for oxidative phosphorylation (15). The 3D structure of this OPPs is composed entirely of $\alpha$-helices joined by connecting loops and is arranged with nine core helices around a large central cavity (13). An elongated hydrophobic tunnel between $\alpha$-helices D and F contains two DDxxD motifs on top for substrate binding and is sealed by F132 at the bottom. Two DDxxD motifs in the amino acid sequences were conserved in all trans-type prenyltransferases (see Figure 1) with the first motif responsible for FPP binding and the second motif for IPP binding (16-19). On the basis of the 3D structure and mutagenesis studies of farnesyl pyrophosphate synthase (another trans-prenyltransferase), a bulky amino acid residue located in the fifth position before the first DDxxD motif of FPPs appeared to block further elongation of the product FPP $(20,21)$. The corresponding amino acid in OPPs is a small amino acid, Ala (Figure 1), which may be required so that the steric obstacle preventing OPPs from synthesizing products larger than FPP could be avoided.

On the basis of the structure of OPPs, we identify several key amino acids which play critical roles in regulating the chain length of the final products. These include Ala76 and Ser77 for $\mathrm{C}_{20}$ production, F132 for the correct product of $\mathrm{C}_{40}$, and Asp62, Ile123, and L128, when mutated to a small Ala, to allow longer products to pass through the bottom of the tunnel.

\section{EXPERIMENTAL PROCEDURES}

Materials. Radiolabeled $\left[{ }^{14} \mathrm{C}\right] \mathrm{IPP}(55 \mathrm{mCi} / \mathrm{mmol})$ was purchased from Amersham Pharmacia Biotech, and FPP was obtained from Sigma. Reverse-phase thin-layer chromatography (TLC) plates were purchased from Merck. PfuTurbo DNA polymerase was obtained from Life Technologies, Inc. The plasmid mini-prep kit, DNA gel extraction kit, and NiNTA resin were purchased from Qiagen. Potato acid phosphatase (2 units/mg) was purchased from Roche Molecular Biochemicals. FXa and the protein expression kit (including the $\mathrm{pET} 32 \mathrm{Xa} / \mathrm{LIC}$ vector and competent JM109 and BL21 cells) were obtained from Novagen. The QuikChange site-directed mutagenesis kit was obtained from Stratagene, Inc. All commercial buffers and reagents were of the highest grade.

Site-Directed Mutagenesis of OPPs. OPPs mutants were prepared by using the QuikChange site-directed mutagenesis kit in conjunction with the Thermotoga OPPs gene template in the $\mathrm{pET} 32 \mathrm{Xa} / \mathrm{Lic}$ vector. The mutagenic primers that were used were prepared by Biobasic Inc. The mutagenic oligonucleotides for performing site-directed mutagenesis are listed in Table 1. The basic procedure of mutagenesis utilizes a supercoiled double-stranded DNA (dsDNA) vector with an insert of interest and two synthetic oligonucleotide primers (forward and backward) containing the desired mutation. The mutation was confirmed by sequencing the entire OPPs mutant gene of the plasmid obtained from the overnight culture. The correct construct was subsequently transformed into Escherichia coli BL21(DE3) for protein expression. The procedure for protein purification followed our reported protocol $(15,22)$. Each purified mutant OPPs was verified by mass spectroscopic analysis, and its purity $(>95 \%)$ was checked by SDS-PAGE.

Crystallization and Data Collection. Five mutants (A76Y, A76Y/S77F, F132A/L128A, F132A/L128A/I123A, and F132A/L128A/I123A/D62A) were crystallized using the hanging drop method from Hampton Research (Laguna Niguel, CA) by mixing $2 \mu \mathrm{L}$ of the OPPs solution ( $10 \mathrm{mg} /$ $\mathrm{mL}$ in $0.1 \%$ Triton $\mathrm{X}-100$ ) with $2 \mu \mathrm{L}$ of the mother liquor [0.1 M Na${ }^{+}$Hepes (pH 7.5), 1.5-2.1 $\mathrm{M} \mathrm{LiSO}_{4}$, and 4-10\% PEG 900], equilibrating with $500 \mu \mathrm{L}$ of the mother liquor. Within 4 days to 2 weeks, crystals had grown to dimensions of $\sim 0.2 \mathrm{~mm} \times \sim 0.2 \mathrm{~mm} \times \sim 0.1 \mathrm{~mm}$. The X-ray diffraction data sets for the A76Y, A76Y/S77F, F132A/L128A, F132A/ L128A/I123A, and F132A/L128A/I123A/D62A were collected to 3.1, 2.7, 3.3, 3.35, and 3.4 Å resolution, respectively. The first data sets of the four mutants were collected in house using a Rigaku MicroMax002 X-ray generator equipped with an R-Axis $\mathrm{IV}^{++}$image plate detector, whereas the F132A/ L128A/I123A/D62A data set was collected at beamline BL17B2 in the National Synchrotron Radiation Research Center (NSSRC, Hsinchu, Taiwan). 

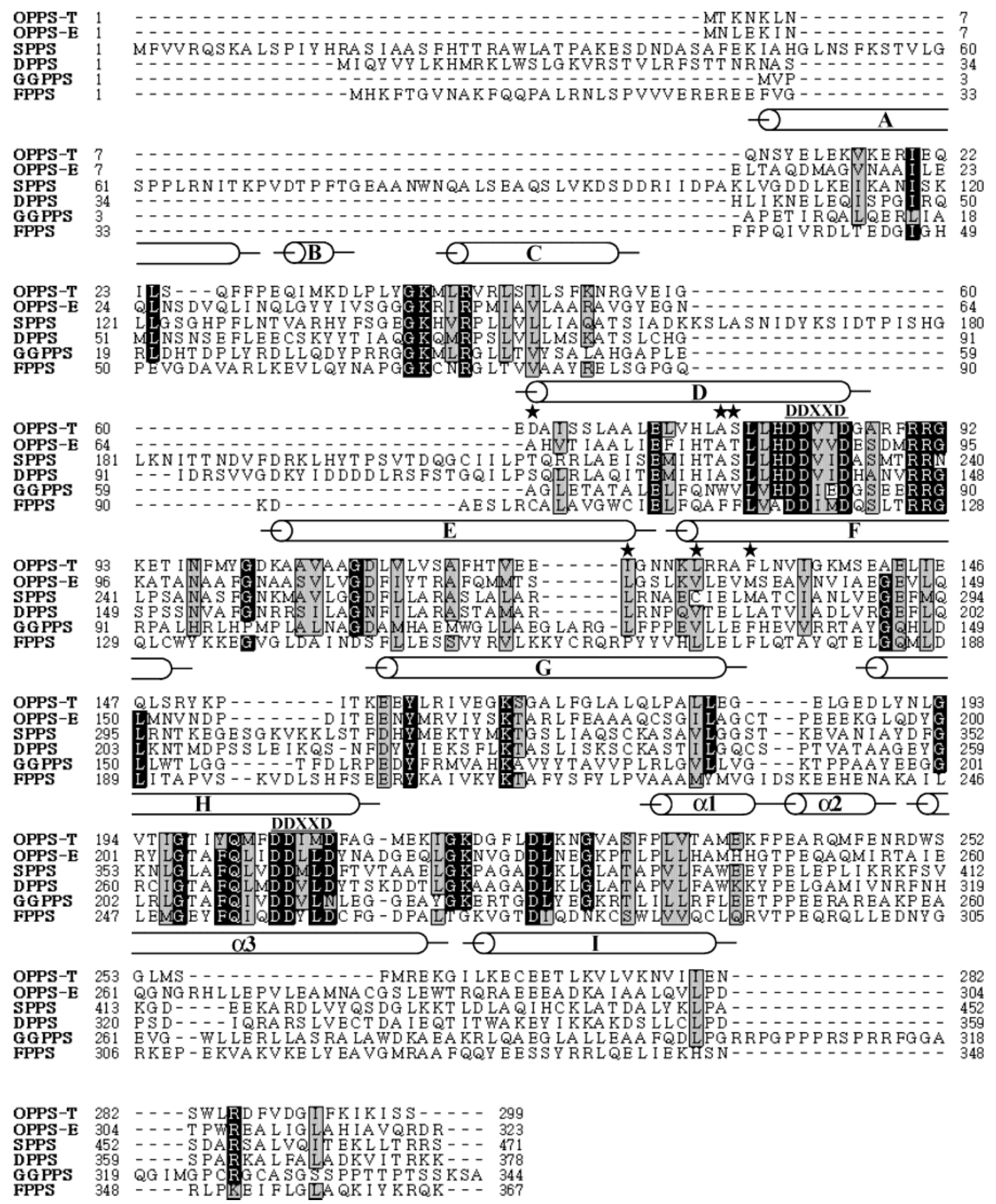

FIGURE 1: Alignment of amino acid sequences of T. maritima OPPs, E. coli OPPs, Mucor $\mathrm{C}_{45}$ SPPs, fission yeast $\mathrm{C}_{50}$ DPPs, Thermus thermophilus $\mathrm{C}_{20}$ GGPPs, and avian FPPs. Two DDXXD motifs are conserved as the substrate binding sites. The stars denote the amino acid residues mutated in this study. A76 and S77 in T. maritima OPPs correspond to the large Y89 and L90 residues in short chain FPPs, respectively. In long chain length enzymes such as $\mathrm{C}_{50}$ decaprenyl pyrophosphate synthase, the corresponding amino acids, Leu, Val, Leu, and Ser, have properties similar to those of F132, L128, I123, and D62 in OPPs, respectively.

The F132A/L128A mutant crystal belongs to tetragonal space group $P 42{ }_{1} 2$ with the following unit cell parameters: $a=b=151.43 \AA$ and $c=67.04 \AA$. Crystals of A76Y, A76Y/S77F, F132A/L128A/I123A, and F132A/L128A/ I123A/D62A belong to tetragonal space group I422 with the following unit cell parameters: $a=b=152.94 \AA$ and $c=$ $65.02 \AA$. All diffraction measurements were carried out on the crystals cryoprotected by adding $10 \%$ glycerol to the reservoir.

Structure Determination and Refinement. The wild-type OPPs structure was determined by the MIR (multiple isomorphous replacement) method using SOLVE (23) as described previously (13). The phases of all OPPs mutant structures were determined by the molecular replacement (MR) method using CNS (24).

Kinetic Parameters for Mutant OPPs. For enzyme activity measurements, each mutant OPPs enzyme was used at a concentration of $0.1 \mu \mathrm{M}$. The reaction was initiated in a 200 $\mu \mathrm{L}$ solution containing $100 \mathrm{mM}$ Hepes ( $\mathrm{pH} 7.5$ ), $5 \mu \mathrm{M}$ FPP, $50 \mu \mathrm{M}\left[{ }^{14} \mathrm{C}\right] \mathrm{IPP}, 50 \mathrm{mM} \mathrm{KCl}$, and $0.5 \mathrm{mM} \mathrm{MgCl}_{2}$ at $25{ }^{\circ} \mathrm{C}$. The enzyme concentration used in all experiments was determined from its absorbance at $280 \mathrm{~nm}$ for each mutant 
Table 2: Data Collection and Refinement Statistics for A76Y, A76Y/S77F, F132A/L128A, F132A/L128A/I123A, and F132A/L128A/I123A/ D62A OPPs ${ }^{a}$

\begin{tabular}{|c|c|c|c|c|c|}
\hline & A76Y & A76Y/S77F & F132A/L128A & F132A/L128A/I123A & F132A/L128A/I123A/D62A \\
\hline space group & I422 & I422 & $P 42_{1} 2$ & I422 & I422 \\
\hline resolution $(\AA)$ & 3.1 & 2.7 & 3.3 & 3.35 & 3.4 \\
\hline \multicolumn{6}{|l|}{ no. of reflections } \\
\hline unique & 7104 & 9589 & 11660 & 5051 & 5575 \\
\hline observed & 71144 & 57001 & 85983 & 28516 & 36078 \\
\hline completeness $(\%)^{b}$ & $99.8(100)$ & $88.4(80.5)$ & $95.1(91.3)$ & $89.0(87.7)$ & $99.8(99.8)$ \\
\hline$R_{\text {merge }}(\%)^{b}$ & $7.1(53.8)$ & $4.7(71.5)$ & $10.0(52.3)$ & $10.0(55.6)$ & $12.0(48.0)$ \\
\hline$I / \sigma\langle I\rangle^{b}$ & $34.89(4.95)$ & $30.31(2.46)$ & $15.05(2.75)$ & $15.51(3.17)$ & $16.78(3.66)$ \\
\hline \multicolumn{6}{|l|}{ refinement } \\
\hline resolution limit $(\AA)$ & $50-3.1$ & $50-2.7$ & $50-3.3$ & $50-3.35$ & $30-3.4$ \\
\hline$R_{\text {factor }}(\%)^{b}$ & $24.43(31.26)$ & $21.98(31.49)$ & $27.70(27.44)$ & $24.07(26.10)$ & $23.58(24.04)$ \\
\hline$R_{\text {free }}(\%)^{b}$ & $27.76(32.52)$ & $28.63(41.21)$ & $32.11(32.57)$ & $29.01(35.59)$ & $28.15(32.49)$ \\
\hline bond lengths $(\AA)$ & 0.0032 & 0.0039 & 0.0041 & 0.0031 & 0.0034 \\
\hline bond angles (deg) & 0.81 & 0.92 & 1.20 & 0.72 & 0.84 \\
\hline average $B$ factors $\left(\AA^{2}\right)$ & 55.5 & 73.5 & 58.4 & 48.2 & 48.2 \\
\hline \multicolumn{6}{|l|}{ Ramachandran plot $(\%)$} \\
\hline favored & 91.2 & 87.6 & 85.4 & 91.6 & 91.6 \\
\hline additionally allowed & 8.4 & 12.4 & 13.8 & 7.6 & 7.6 \\
\hline generously allowed & 0.4 & 0 & 0.8 & 0.8 & 0.8 \\
\hline disallowed & 0 & 0 & 0 & 0 & 0 \\
\hline
\end{tabular}

${ }^{a}$ The unit cell dimensions for F132A/L128A (P4212) are as follows: $a=b=151.43 \AA$ and $c=67.04 \AA$ (two molecules per asymmetric unit). For A76Y, A76Y/S77F, F132A/L128A/I123A, and F132A/L128A/I123A/D62A (I422) they are as follows: $a=b=152.94 \AA$ and $c=65.02 \AA$ (one molecule per asymmetric unit). ${ }^{b}$ The values in parentheses are for highest-resolution shells.

OPPs. The reaction was terminated by adding $10 \mathrm{mM}$ (final concentration) EDTA, and the product was extracted with 1-butanol. The product was quantitated by counting the radioactivity in the butanol phase $\left(\left[{ }^{14} \mathrm{C}\right] \mathrm{IPP}\right.$ in the aqueous phase) using a Beckman LS6500 scintillation counter. The steady-state $k_{\text {cat }}$ values of the mutant enzymes were calculated on the basis of the initial rate of IPP consumption.

Product Analysis. The OPPs reaction with the mixture containing $0.5 \mu \mathrm{M}$ enzyme (wild-type and mutant OPPs), $250 \mu \mathrm{M}\left[{ }^{14} \mathrm{C}\right] \mathrm{IPP}, 5 \mu \mathrm{M}$ FPP, $0.1 \%$ Triton $\mathrm{X}-100,0.5 \mathrm{mM}$ $\mathrm{MgCl}_{2}$, and $50 \mathrm{mM} \mathrm{KCl}$ in $100 \mathrm{mM}$ Hepes buffer ( $\left.\mathrm{pH} 7.5\right)$ was performed for $72 \mathrm{~h}$ at $25^{\circ} \mathrm{C}$. The [FPP]:[IPP] ratio was extended to 1:50 compared with the ratio of 1:10 used in the previous study (13) since the longer products generated by the mutants in the study presented here required more IPP. A higher ratio (i.e., 1:100) did not further elongate the product chain lengths (data not shown). The $10 \mathrm{mM}$ EDTA was used to terminate the reactions. The radiolabeled polyprenyl pyrophosphate products were extracted with 1-butanol. The 1-butanol was then evaporated, and the $20 \%$ propanol solution containing 4.4 units $/ \mathrm{mL}$ acidic phosphatase, $0.1 \%$ Triton, and $50 \mathrm{mM}$ sodium acetate $(\mathrm{pH} 4.7)$ was prepared to convert polyprenyl pyrophosphate products to corresponding alcohols according to the reported procedure (25). After the pyrophosphate hydrolysis catalyzed by acidic phosphatase was completed, the polyprenols were extracted with $n$-hexane. The hexane volume was reduced by evaporation. The polyprenols were separated by reversed-phase TLC using a high (39:1) acetone:water ratio as the mobile phase. The content of organic acetone which was higher than that previously used (19:1) was needed for longer products (13). The radiolabeled products were identified by autoradiography using a FUJIFILM BAS-1500 bioimaging analyzer according to their reported $R_{f}$ values. The percentages of the intermediates and product formed in the OPPs reaction were calculated from the measured intensities normalized by the numbers of $\left[{ }^{14} \mathrm{C}\right] \mathrm{IPP}$ incorporated.

\section{RESULTS}

Crystal Structures of the OPPs Mutants. The wild-type and F132A OPPs crystal structures have been previously reported (13). In the study presented here, we have generated three new mutants, F132A/L128A, F132A/L128A/I123A, and F132A/L128A/I123A/D62A, and determined their crystal structures. Moreover, we also determined the structures of S77F and F76Y/S77F which were prepared in our previous study (13). Among the five OPP mutants, F132A/L128A OPPs belongs to the $P 42_{1} 2$ space group. Each asymmetric unit of the crystal unit cell contains one OPPs dimer, which is the active form of the enzyme. On the other hand, A76Y, A76Y/S77F, F132A/L128A/I123A, and F132A/L128A/ I123A/D62A belong to the I422 space group. A crystallographic 2-fold axis is coincident with the dyad of the dimer, and each asymmetric unit of the crystal unit cell contains only one OPPs monomer. Data collection and refinement statistics for the structural analysis of A76Y, A76Y/S77F, F132A/L128A, F132A/L128A/I123A, and F132A/L128A/ I123A/D62A OPPs are summarized in Table 2. Like wildtype OPPs, all mutant structures contain $12 \alpha$-helices, nine of them surrounding a large central cavity (helices A-I) and an elongated tunnel-shaped active site cavity surrounded by four $\alpha$-helices (helices C, D, F, and H) (Figure 2A). Two DDxxD motifs for substrate binding are located in helices $\mathrm{D}$ and $\mathrm{H}$ near the top of the tunnel. At the bottom of helix F, F132 provides the floor to prevent extra chain elongation of the OPP product (13). Amino acids L128, I123, and D62 underneath F132 may form additional seals as shown by the side view and bottom-up stereoview of the tunnel in the wildtype OPPs crystal structure (Figure 2B,C). When those four amino acids were replaced with Ala, the F132A/L128A/ I123A/D62A mutant shows a hollow tunnel with open space at the bottom of the tunnel as shown by the side view and the bottom-up stereoview (Figure 2B,D). The other mutants, F132A/L128A and F132A/L128A/I123A, exhibit a partially blocked tunnel (data not shown). 

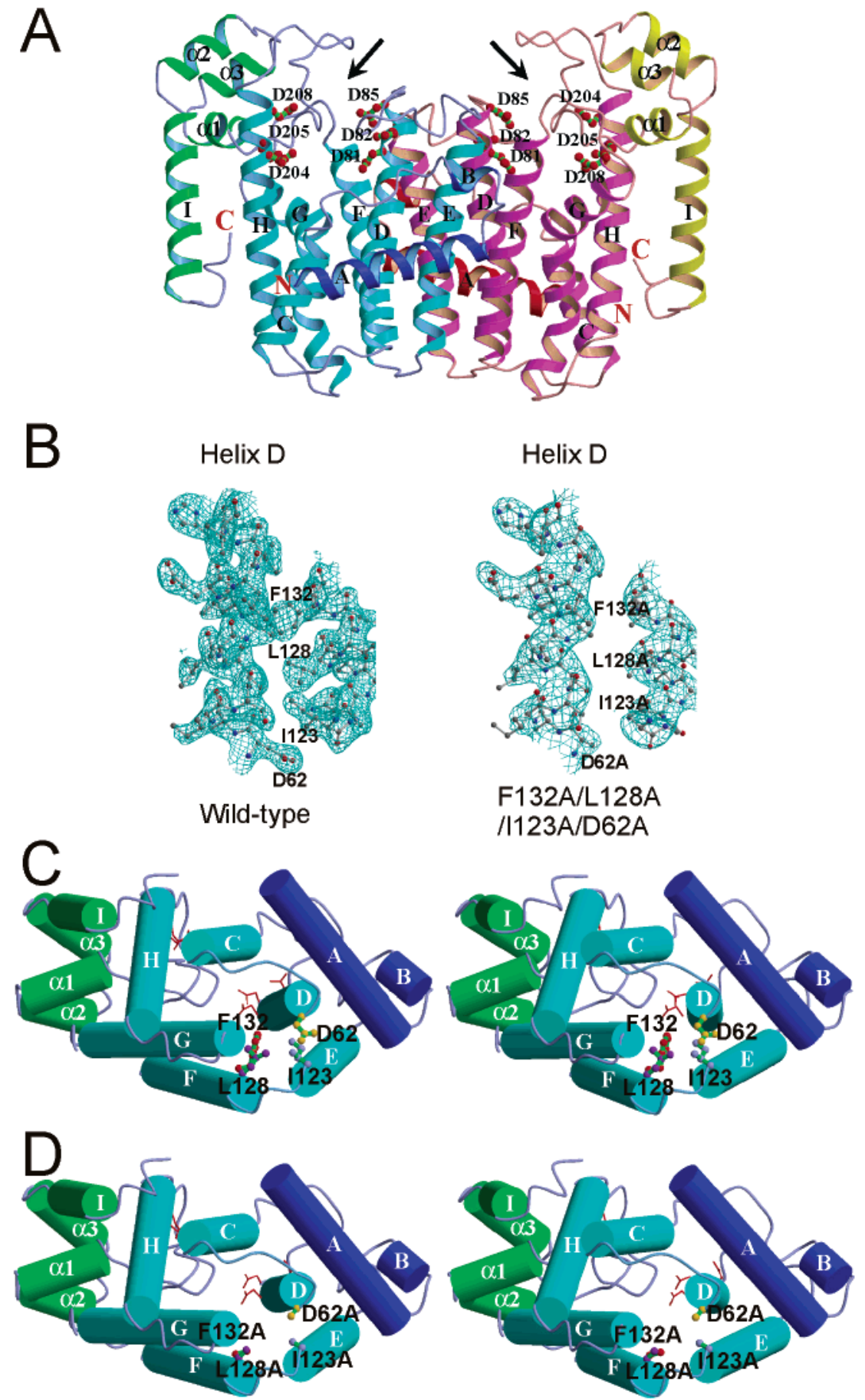

FIGURE 2: Crystal structures of wild-type and F132A/L128A/I123A/D62A mutant OPPs. In panel A, the dimeric wild-type OPPs crystal structure is shown as a ribbon diagram. Two monomeric subunits are shown in blue and purple. Arrows indicate the active site locations where Asp-rich motifs are responsible for FPP and IPP binding. In panel B, the side views of the active site tunnels in wild-type and mutant OPPs are compared. The mutant apparently has an unblocked internal space that originated from the removal of the side chains. Panels $\mathrm{C}$ and D show the bottom-up stereoview structures of wild-type OPPs and the mutant, respectively. Four amino acids (F132, L128, I123, and D62) occupy the central area at the bottom of the tunnel. The internal part of tunnel becomes clear after these amino acids are replaced with the small Ala residue.

Products Synthesized by F132A/L128A, F132A/L128A/ I123A, and F132A/L128A/I123A/D62A. By removing the blockade along the pathway of product chain elongation, we aimed to engineer mutant enzymes which can produce a product much larger than $\mathrm{C}_{40} \mathrm{OPP}$. We thus produced three OPPs mutants (F132A/L128A, F132A/L128A/I123A, and F132A/L128A/I123A/D62A). These enzymes exhibit slightly lower activities than the wild type (Table 3), so the time for obtaining the final products was adjusted according to the relative activity of each mutant. Compared to the wild-type OPPs and the F132A mutant which synthesize $\mathrm{C}_{40}$ and $\mathrm{C}_{50}$ as the major products, respectively (lanes 1 and 2 of Figure 3), F132A/L128A (lane 3), F132A/L128A/I123A (lane 4), and $\mathrm{F} 132 \mathrm{~A} / \mathrm{L} 128 \mathrm{~A} / \mathrm{I} 123 \mathrm{~A} / \mathrm{D} 62 \mathrm{~A}$ (lane 5) produce $\mathrm{C}_{60}$ as the major product $(30,38$, and $21 \%$ for the latter three mutants, respectively). Finally, the quadruple mutant synthesizes an up to $\mathrm{C}_{95}$ product. Apparently, step-by-step removal of the large amino acids near the bottom of the tunnel gradually 
Table 3: Steady-State Activities of T. maritima OPPs (wild type and mutants) Assayed under $5 \mu \mathrm{M}$ FPP and $50 \mu \mathrm{M}\left[{ }^{14} \mathrm{C}\right] \mathrm{IPP}$ at $\mathrm{pH}$ 7.5 and $25^{\circ} \mathrm{C}$

\begin{tabular}{lc}
\hline \multicolumn{1}{c}{ T. maritima OPPs } & steady-state activity $\left(\mathrm{s}^{-1}\right)$ \\
\hline wild type & $5.00 \times 10^{-3 a}$ \\
A76Y & $7.30 \times 10^{-5 a}$ \\
S77F & $2.91 \times 10^{-7 a}$ \\
A76Y/S77F & $2.26 \times 10^{-7 a}$ \\
F132A & $5.09 \times 10^{-3 a}$ \\
F132A/L128A & $8.03 \times 10^{-4}$ \\
F132A/L128A/I123A & $6.58 \times 10^{-4}$ \\
F132A/L128A/I123A/D62A & $6.10 \times 10^{-4}$ \\
\hline${ }^{a}$ The numbers are taken from ref 13. &
\end{tabular}

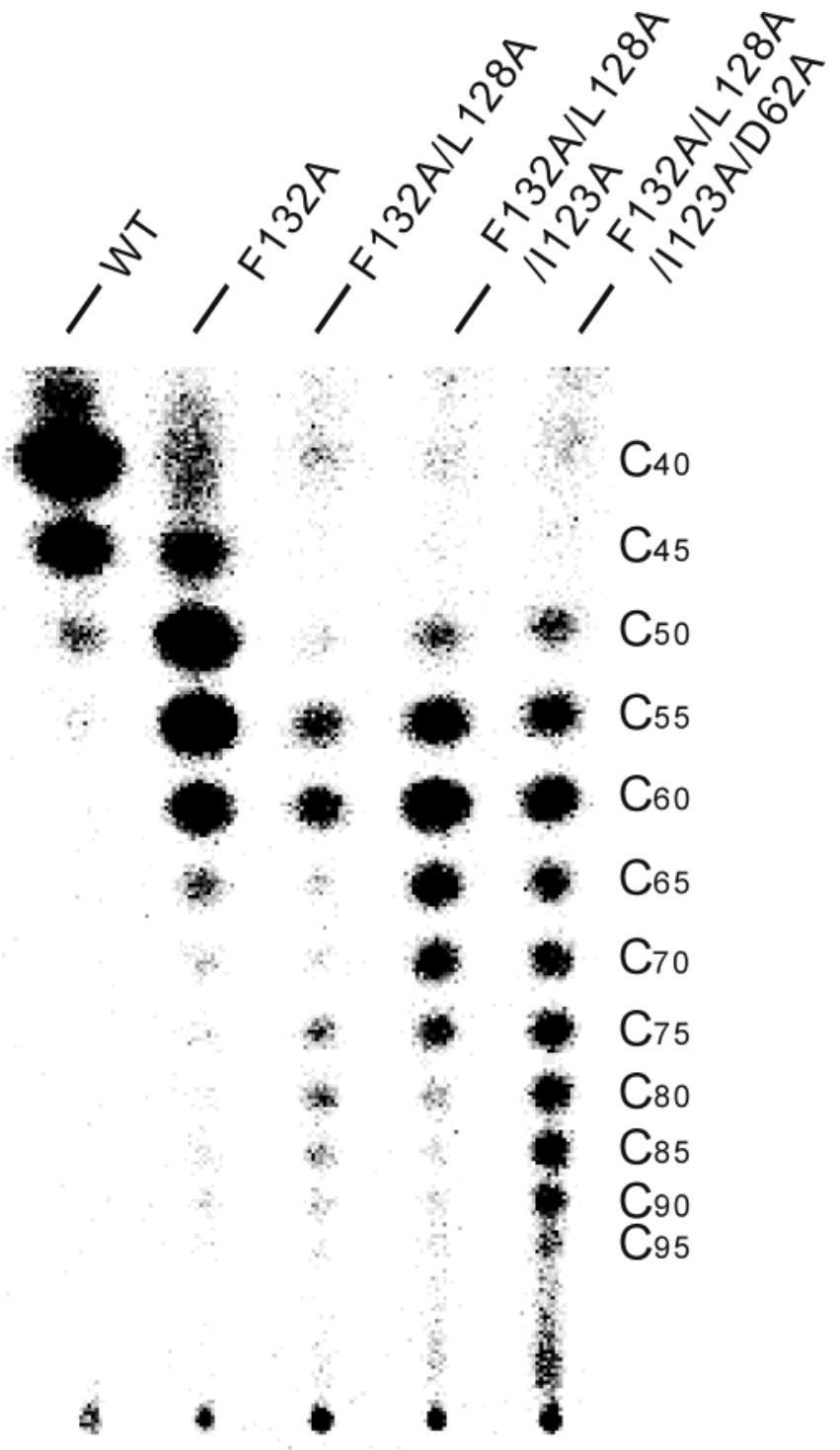

FIGURE 3: Products synthesized by wild-type, F132A, F132A/ L128A, F132A/L128A/I123A, and F132A/L128A/I123A/D62A OPPs. The reaction mixture containing each mutant enzyme at 0.5 $\mu \mathrm{M}, 5 \mu \mathrm{M}$ FPP, and $250 \mu \mathrm{M}\left[{ }^{14} \mathrm{C}\right] \mathrm{IPP}$ was incubated at $25^{\circ} \mathrm{C}$ for $72 \mathrm{~h}$ to complete the reaction. The final products were extracted and analyzed using TLC and a phosphorimager. Among the mutants, F132A/L128A/I123A/D62A synthesizes the largest products (the longest product being $\mathrm{C}_{95}$ ). Some products larger than $\mathrm{C}_{95}$ seem to exist (close to the starting position), but they were not identified unequivocally.

increases the chain lengths of the final products of the mutants. However, the $\mathrm{C}_{60}$ major product shared by F132A/

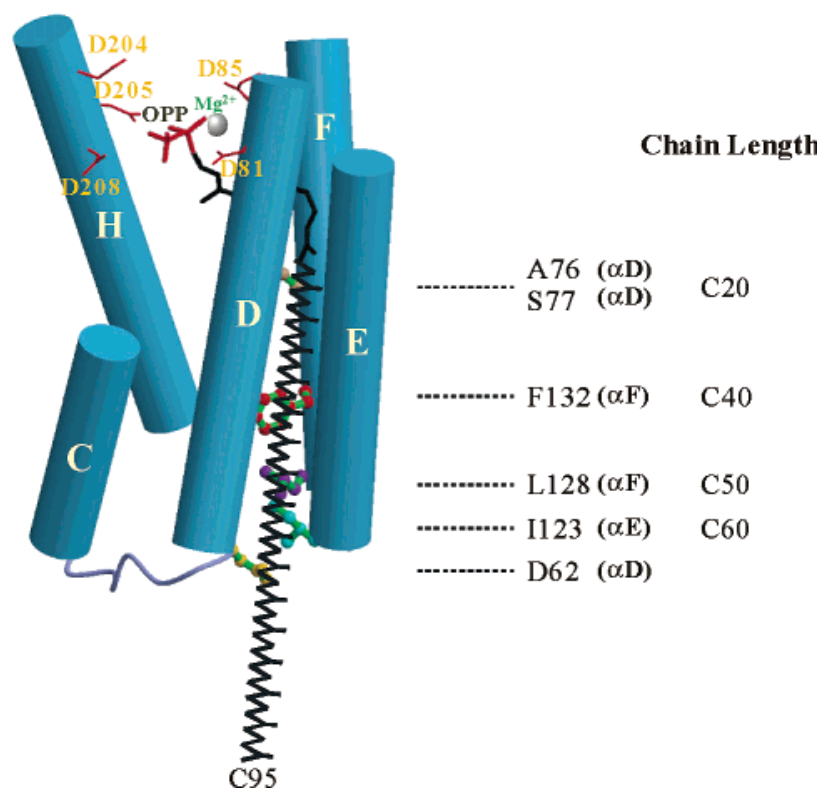

FIGURE 4: Proposed molecular ruler for chain length determination catalyzed by OPPs. The first DDxxD motif attached to helix D represents the FPP binding site. S77 and A76 are located in immediate neighborhood below FPP. Their substitution with large amino acids led to the formation of $\mathrm{C}_{20}$ as a final product. F132, L128, and I123 are located on the bottom of helix D to block the chain elongation of $\mathrm{C}_{40}, \mathrm{C}_{50}$, and $\mathrm{C}_{60}$, respectively.

L128A, F132A/L128A/I123A, and F132A/L128A/I123A/ D62A indicates that the chain of $\mathrm{C}_{60}$ may reach the location of I123, and the I123A mutation is not able to create a sufficiently large opening to allow further chain elongation effectively. The replacement of I123 with an even smaller Gly residue in the mutant F132A/L128A/I123G/D62A still failed to provide sufficient space to efficiently "thread" the growing isoprenoid, and the major product remained $\mathrm{C}_{60}$ (data not shown). Indeed, the diameter of the tunnel in the section of I123A is $\sim 5.2 \AA$, the smallest among the areas beside the four amino acids being mutated to Ala. Therefore, when F132 is converted to Ala, the product chain length is extended from the wild-type $\mathrm{C}_{40}$ to $\mathrm{C}_{50}$ for the mutant. With the substitution of L128 with Ala in addition to the F132A mutation, the major product becomes $\mathrm{C}_{60}$. But further change to I123A fails to extend the chain length of the major product. On the basis of these results, a molecular ruler for the chain elongation reactions catalyzed by OPPs is proposed, which shows that F132, L128, and I123 are located at the $\mathrm{C}_{40}, \mathrm{C}_{50}$, and $\mathrm{C}_{60}$ key positions, respectively (Figure 4 ). The position of L128 is a bit lower, so a significant amount of $\mathrm{C}_{55}$ was also produced along with $\mathrm{C}_{50}\left(37: 29 \mathrm{C}_{50}\right.$ : $\mathrm{C}_{55}$ ratio, lane 2 of Figure 3).

Crystal Structures of $A 76 Y$ and A76Y/S77F. Two sulfate ions (pyrophosphate-like) bound to the two DDxxD Asprich motifs via $\mathrm{Mg}^{2+}$ represent the substrate binding locations of wild-type OPPs (13). The two mutant amino acids, A76Y and S77F, are located approximately $10-13 \AA$ beneath the first DDxxD motif, allowing adequate space for accommodation of a FPP molecule. We have shown that the A76Y and $\mathrm{A} 76 \mathrm{Y} / \mathrm{S} 77 \mathrm{~F}$ mutants produced $\mathrm{C}_{20}$ since the substituted bulky residues block the continuous chain elongation of $\mathrm{C}_{20}$. Surprisingly, the single mutant S77F generates $\mathrm{C}_{20}$ in much smaller quantities than the double mutant A76Y/S77F (13). S77F has an activity similar to that of A76Y/S77F, although 

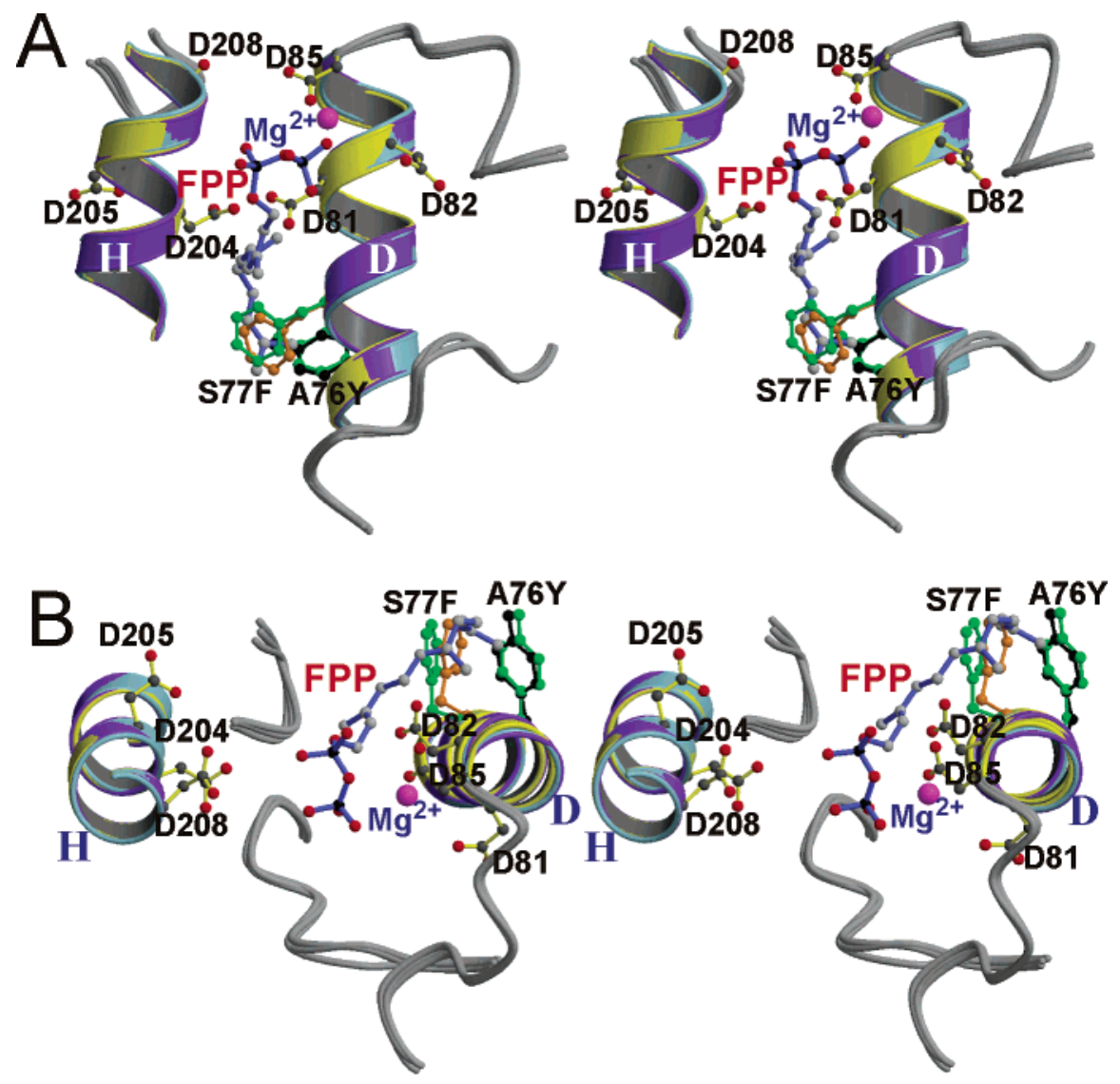

FIGURE 5: Structures of A76Y, S77F, and A76Y/S77F mutants. The side view (A) and the top view (B) of the A76Y and S77F structures superimposed with the A76Y/S77F structure are shown using a ribbon diagram. The A76Y structure is shown in blue, the S77F structure in yellow, and the A76Y/S77F structure in indigo. The residues of A76Y, S77F, and A76Y/S77F are shown in black, orange, and green, respectively. The modeled FPP is placed in the active site with the pyrophosphate oxyanion (in red) directed to the bound $\mathrm{Mg}^{2+}$ (purple dot) which is coordinated with the first DDxxD motif, and the hydrocarbon tail is pointed to the aromatic side chain of the mutated residues. In A76Y/S77F, the bulky Y76 pushes F77 away from its original place where the FPP chain elongation is partially blocked.

their $k_{\text {cat }}$ values are significantly smaller than that of the wild type (Table 3). This seems contradictory since the replacement of two small amino acids, Ala and Ser, with large residues, Tyr and Phe, respectively, simultaneously should provide a larger seal for blocking the chain elongation than does the S77F single mutation. On the other hand, A76Y also produced a significant amount of $\mathrm{C}_{20}$ as the final product (13). This mutant has a $k_{\text {cat }}$ value similar to that of the wild type, but much larger than the $k_{\text {cat }}$ values of S77F and A76Y/ S77F (Table 3).

The structures of S77F and A76Y/S77F determined in this study provide a possible answer to the above contradiction. The structure of A76Y/S77F shows that the large Y76 pushes F77 away and leaves a space between them, thereby allowing the FPP hydrocarbon terminus to wedge between the two aromatic side chains (see the green benzene rings for Y76 and F77 as shown in Figure 5). On the other hand, the F77 aromatic side chain (the orange benzene ring) of S77F is directly underneath the FPP hydrocarbon tail and could block the FPP chain elongation more effectively so that less $C_{20}$ is produced. The previously determined structure of A76Y (13) shows that Y76 (the black benzene ring) alone could not completely stop the FPP elongation so a larger amount of $\mathrm{C}_{20}$ was obtained with this mutant. In the molecular ruler shown in Figure 4, we could add A76 and S77 at the stage of $\mathrm{C}_{20}$.

\section{DISCUSSION}

We previously hypothesized that the substitution of the large F132 with a smaller Ala removes the floor of the tunnel, thereby allowing the formation of products $\left(\mathrm{C}_{50}\right)$ longer than the $\mathrm{C}_{40}$ produced by the wild type (13). From the side view of the tunnel (Figure 2B), F132 is well positioned to seal the bottom of the tunnel. Beneath F132, there are several additional amino acids (L128, I123, and D62) which may interfere with further chain elongation of the products. In this study, we have generated the F132A/L128A, F132A/ L128A/I123A, and F132A/L128A/I123A/D62A mutants in addition to previously generated F132A to examine their products. The product can be extended from $\mathrm{C}_{40}$ to finally $\mathrm{C}_{95}$ for the quadruple mutant. The results have been rationalized by the crystal structures of these mutants showing that the mutants have a more open cavity at the bottom of the tunnel to allow the extra chain elongation to penetrate. By comparing the amino acid sequence of $T$. maritima OPPs with the sequences of other trans-prenyltransferases (Figure 1), we find that the amino acids corresponding to F132, L128, I123, and D62 of OPPs are Leu, Ala, Ser, and Gly, respectively, in short chain length FPPs. There are no particular requirements for these amino acids (large or small, hydrophobic or hydrophilic) since these residues are located out of the active site for the short chain length enzymes. 
However, in the long chain length enzymes such as decaprenyl pyrophosphate synthase making the $\mathrm{C}_{50}$ product, the corresponding amino acids are Leu, Val, Leu, and Ser, with properties similar to those of F132, L128, I123, and D62 in OPPs, respectively. Our results here thus provide a model for other trans-prenyltransferases in understanding the mechanism of chain elongation. In OPPs, a molecular ruler is defined as shown in Figure 4.

From the crystal structure of OPPs, A76 and S77 are right below the first DDxxD motif where FPP is bound. A76 and S77 are amino acids located at the fourth and fifth positions, respectively, upstream from the first DDxxD motif in the OPPs sequence (see Figure 1). When these small amino acids were substituted with bulky Tyr and Phe, the final products of the mutant OPPs became $\mathrm{C}_{20}$, suggesting the role of the steric hindrance of the large amino acids at these positions for substrate chain elongation. Moreover, it is notable that the crystal structures of $\mathrm{A} 76 \mathrm{Y}$ and $\mathrm{A} 76 \mathrm{Y} / \mathrm{S} 77 \mathrm{~F}$ remain dimeric in contrast to the previous report that the A79Y mutation in E. coli OPPs (corresponding to T. maritima A76Y) affects the dimer formation (26). The corresponding residues at these positions for FPPs from Bacillus stearothermophilus are larger Tyr and Leu, respectively. When the Tyr (Y81) was changed to a smaller residue such as Gly or Ala, the product chain length was increased at most to $\mathrm{C}_{30}$, consistent with a "molecular ruler" mechanism (27). In the context of lipid selectivity, a molecular ruler hypothesis was proposed for protein farnesyltransferase, and the mutational studies have been carried out with protein geranylgeranyltransferase to identify the specific residues that determine prenyl group specificity $(28,29)$. Thus, the molecular ruler concept is a general one adopted by enzymes that synthesize or transfer hydrocarbons of various chain lengths.

In T. maritima OPPs, the quadruple mutant can generate the $\mathrm{C}_{95}$ product, a record high for trans-prenyltransferases, but the chain length is still much shorter than that of the polymers containing thousands of IPP units produced by the cis-type rubber prenyltransferases. The enlargement on the side opening may be required in addition to the bottom opening of the tunnel to produce super long trans-type polymers. Very long chain polymers may be obtained by adding some biological accessory apparatus to capture the huge hydrophobic products.

\section{ACKNOWLEDGMENT}

We thank Dr. Shuenn-Shing Chern for his help in data collection. The synchrotron data collection was conducted with the Biological Crystallography Facilities (beamline BL17B2 at NSSRC) supported by the National Science Council (NSC).

\section{REFERENCES}

1. Liang, P. H., Ko, T. P., and Wang, A. H.-J. (2002) Structure, mechanism, and function of prenyltransferases, Eur. J. Biochem. 269, 3339-3354.

2. Ogura, K., and Koyama, T. (1998) Enzymatic aspects of isoprenoid chain elongation, Chem. Rev. 98, 1263-1276.

3. Chen, A. P.-C., Chen, Y. H., Liu, H. P., Li, Y. C., Chen, C. T., and Liang, P. H. (2002) Synthesis and application of a fluorescent substrate analogue to study ligand interactions for undecaprenyl pyrophosphate synthase, J. Am. Chem. Soc. 124, 15217-15224.

4. Oh, S. K., Han, K.-H., Ryu, S. B., and Kang, H. (2000) Molecular cloning, expression, and functional analysis of a cis-prenyltransferase from Arabidopsis thaliana, J. Biol. Chem. 275, 1848218488 .
5. Sato, M., Sato, K., Nishikawa, S.-I., Hirata, A., Kato, J.-I., and Nakano, A. (1999) The yeast RER2 gene, identified by endoplasmic reticulum protein localization mutations, encodes cis-prenyltransferase, a key enzyme in dolichol synthesis, Mol. Cell. Biol. $19,471-483$.

6. Cornisk, K. (2001) Similarities and differences in rubber biochemistry among plant species, Phytochemistry 57, 1123-1134.

7. Asawatreratanakul, K., Zhang, Y.-W., Wititsuwannakul, D., Wititsuwannakul, R., Takahashi, S., Rattanapittayaporn, A., and Koyama, T. (2003) Molecular cloning, expression and characterization of cDNA encoding cis-prenyltransferases from Hevea brasiliensis. A key factor participating in natural rubber biosynthesis, Eur. J. Biochem. 270, 4671-4680.

8. Ishii, K., Sagami, H., and Ogura, K. (1983) Decaprenyl pyrophosphate synthetase from mitochondria of pig liver, Biochem. Biophys. Res. Commun. 116, 500-506.

9. Ko, T. P., Chen, Y. K., Robinson, H., Tsai, P. C., Gao, Y.-G. Chen, A. P.-C., Wang, A. H.-J., and Liang, P. H. (2001) Mechanism of product chain length determination and the role of a flexible loop in Escherichia coli undecaprenyl-pyrophosphate synthase catalysis, J. Biol. Chem. 276, 47474-47482.

10. Chen, Y. H., Chen, A. P., Chen, C. T., Wang, A. H.-J., and Liang, P. H. (2002) Probing the conformational change of Escherichia coli undecaprenyl pyrophosphate synthase during catalysis using an inhibitor and tryptophan mutants, J. Biol. Chem. 277, 73697376.

11. Chang, S. Y., Ko, T. P., Liang, P. H., and Wang, A. H.-J. (2003) Catalytic mechanism revealed by the crystal structure of undecaprenyl pyrophosphate synthase in complex with sulfate, magnesium, and Triton, J. Biol. Chem. 278, 29298-29307.

12. Guo, R. T., Ko, T. P., Chou, C. C., Shr, H. L., Chu, H. M., Tsai, Y. H., Liang, P. H., and Wang, A. H.-J. (2003) Preliminary X-ray diffraction analysis of octaprenyl pyrophosphate synthase crystals from Thermotoga maritima and Escherichia coli, Acta Crystallogr. D59, 2265-2268.

13. Guo, R. T., Kuo, C. J., Chou, C. C., Ko, T. P., Shr, H. L., Liang, P. H., and Wang, A. H.-J. (2004) Crystal structure of octaprenyl pyrophosphate synthase from hyperthermophilic Thermotoga maritima and mechanism of product chain length determination, J. Biol. Chem. 279, 4903-4912.

14. Chen, A., Kroon, P. A., and Poulter, C. D. (1994) Isoprenyl diphosphate synthases: protein sequence comparisons, a phylogenetic tree, and predictions of secondary structure, Protein Sci. $3,600-607$.

15. Kuo, T. H., and Liang, P. H. (2002) Reaction kinetic pathway of the recombinant octaprenyl pyrophosphate synthase from Thermotoga maritima: how is it different from that of the mesophilic enzyme, Biochim. Biophys. Acta 1599, 125-133.

16. Marrero, P. F., Poulter, C. D., and Edwards, P. A. (1992) Effects of site-directed mutagenesis of the highly conserved aspartate residues in domain II of farnesyl diphosphate synthase activity, J. Biol. Chem. 267, 21873-21878.

17. Joly, A., and Edwards, P. A. (1993) Effect of site-directed mutagenesis of conserved aspartate and arginine residues upon farnesyl diphosphate synthase activity, J. Biol. Chem. 268, 2698326989.

18. Koyama, T., Tajima, M., Sano, H., Doi, T., Koike-Takeshita, A., Obata, S., Nishino, T., and Ogura, K. (1996) Identification of significant residues in the substrate binding site of Bacillus stearothermophilus farnesyl diphosphate synthase, Biochemistry $35,9533-9538$.

19. Song, L., and Poulter, C. D. (1994) Yeast farnesyl diphosphate synthase: site-directed mutagenesis of residues in highly conserved prenyltransferase domain I and II, Proc. Natl. Acad. Sci. U.S.A. 91, 3044-3048.

20. Tarshis, L. C., Yan, M., Poulter, C. D., and Sacchettini, J. C. (1994) Crystal structure of recombinant farnesyl diphosphate synthase at $2.6 \AA$ resolution, Biochemistry 33, 10871-10877.

21. Tarshis, L. C., Proteau, P. J., Kellogg, B. A., Sacchettini, J. C., and Poulter, C. D. (1996) Regulation of product chain length by isoprenyl diphosphate synthase, Proc. Natl. Acad. Sci. U.S.A. 93, 15018-15023

22. Pan, J. J., Kuo, T. H., Chen, Y. K., Yang, L. W., and Liang, P. H. (2002) Insight into the activation mechanism of Escherichia coli octaprenyl pyrophosphate synthase derived from pre-steady-state kinetic analysis, Biochim. Biophys. Acta 1594, 64-73.

23. Terwilliger, T. C., and Berendzen, J. (1999) Automated MAD and MIR structure solution, Acta Crystallogr. D55, 849-861. 
24. Brunger, A. T., Adams, P. D., Clore, G. M., DeLano, W. L., Gros, P., GrosseKunstleve, R. W., Jiang, J.-S., Kuszewski, J., Nilges, M., Pannu, N. S., Read, R. J., Rice, L. M., Simonson, T., and Warren, G. L. (1998) Crystallography \& NMR system: A new software suite for macromolecular structure determination, Acta Crystallogr. D54, 905-921.

25. Pan, J. J., Chiou, S. T., and Liang, P. H. (2000) Product distribution and pre-steady-state kinetic analysis of Escherichia coli undecaprenyl pyrophosphate synthase reaction, Biochemistry 39, 1093610942.

26. Kainou, T., Okada, K., Suzuki, K., Nakagawa, T., Matsuda, H., and Kawamukai, M. (2001) Dimer formation of octaprenyldiphosphate synthase (IspB) is essential for chain length determination of ubiquinone, J. Biol. Chem. 276, 7076-7883.
27. Ohnuma, S.-I., Narita, K., Nakazawa, T., Ishida, C., Takeuchi, Y., Ohto, C., and Nishito, T. (1996) A role of the amino acid residue located on the fifth position before the first aspartate-rich motif of farnesyl diphosphate synthase on determination of the final product, J. Biol. Chem. 271, 30748-30754.

28. Long, S. B., Casey, P. J., and Beese, L. S. (1998) Cocrystal structure of protein farnesyltransferase complexed with a farnesyl diphosphate substrate, Biochemistry 37, 9612-9618.

29. Taylor, J. S., Reid, T. S., Terry, K. L., Casey, P. J., and Beese, L. S. (2003) Structure of mammalian protein geranylgeranyltransferase type-I, EMBO J. 22, 5963-5974.

BI036336D 